\title{
O SECRETÁRIO EXECUTIVO E A TECNOLOGIA DA INFORMAÇÃO: UM ESTUDO SOBRE A UTILIZAÇÃO DE RECURSOS TECNOLÓGICOS PELOS PROFISSIONAIS DA CIDADE DE BELÉM/PA.
}

THE EXECUTIVE SECRETARY AND THE INFORMATION TECHNOLOGY: A STUDY ABOUT THE USE OF TECHNOLOGICAL RESOURCES BY PROFESSIONALS IN THE CITY OF BELÉM/PA

\author{
Marco Antonio Lima \\ Doutor em Desenvolvimento Socioambiental pela Universidade Federal do Pará - UFPA. \\ Professor do Mestrado em Administração da Universidade da Amazônia - PPGA/UNAMA. \\ E-mail: adm.marcoantonio@bol.com.br (Brasil)
}

\footnotetext{
Alessandra de Paula Lima Soares

Bacharel em Secretariado Executivo Trilíngue pela Universidade Federal do Pará - UFPA.

E-mail: alessandra_limares@hotmail.com (Brasil)
} 


\title{
O SECRETÁRIO EXECUTIVO E A TECNOLOGIA DA INFORMAÇÃO: UM ESTUDO
} SOBRE A UTILIZAÇÃO DE RECURSOS TECNOLÓGICOS PELOS PROFISSIONAIS DA CIDADE DE BELÉM/PA.

\section{RESUMO}

Este estudo tem como objetivo analisar o grau de intensidade de utilização de aplicativos de Tecnologia da Informação (TI) por profissionais de Secretariado Executivo em organizações estabelecidas na cidade de Belém, no estado do Pará. Com a finalidade de sistematização da pesquisa, os aplicativos foram classificados em quatro diferentes categorias (Automação de Escritório, Comunicação, Gestão de Processos e Softwares Empresariais), baseados em uma adaptação do modelo de Turban, Mclean e Wetherbe (2004). Foi realizada aplicação de questionários a uma amostra não probabilística acidental composta por 35 profissionais graduados dos cursos de Bacharelado em Secretariado Bilíngue e Trilíngue, residentes na cidade de Belém/PA. O modelo analítico foi baseado na análise estatística descritiva e análise de cluster, obtendo como principais resultados a identificação dos índices de utilização dos recursos tecnológicos em escala coletiva e individual; e a classificação dos indivíduos em três agrupamentos com diferentes perfis de utilização dos recursos. Concluiu-se que os profissionais pesquisados utilizam os recursos tecnológicos disponibilizados de forma desproporcional, concentrando-se na utilização de aplicativos para processamento de textos e para comunicação eletrônica, desperdiçando o potencial de ferramentas importantes como os aplicativos para gerenciamento de bancos de dados e gestão de projetos, que registraram baixíssimos índices de utilização nesta pesquisa.

Palavras-chave: Tecnologia da Informação; Recursos e Sistemas de Informação; Secretariado Executivo.

THE EXECUTIVE SECRETARY AND THE INFORMATION TECHNOLOGY: A STUDY

\section{ABOUT THE USE OF TECHNOLOGICAL RESOURCES BY PROFESSIONALS IN THE CITY OF BELÉM/PA}

\begin{abstract}
This study aims to analyze the degree of intensity in the use of information technology (IT) applications by Executive Secretariat professionals in organizations established in the city of Belém, in Pará State In order to systematize this research, the applications were classified into four different categories (Office Automation, Communication, Process Management and Enterprise Software), based on an adaptation of the model of Turban, Mclean and Wetherbe (2004). Questionnaires to a non-probability accidental sample composed of 35 professionals graduates in the Bilingual and Trilingual Executive Secretariat undregraduate programas were carried out, who were also living in the city of Belém/PA. The analytical model was based on descriptive statistical analysis and cluster analysis, obtaining the main results of the identification rates of use of technological resources in individual and collective scale, and the classification of individuals into three groups with different profiles of resources use. It was concluded that the surveyed professionals use the technological resources available disproportionately focusing on office applications for word processing and electronic communication, wasting potential applications as important tools for managing databases and management projects, which recorded low rates of use in this research.
\end{abstract}

Keywords: Information Technology; Resources and Information Systems; Executive Secretariat.

Revista de Gestão e Secretariado - GeSec, São Paulo, v. 5, n. 2, p 138-157, mai./ago. 2014. 


\section{INTRODUÇÃO}

A Tecnologia da Informação (TI) passou a ser vista, mais intensamente nas últimas décadas, como uma peça fundamental dentro das organizações, por estar o mercado cada vez mais instável e competitivo. Isto acaba obrigando as empresas a recorrerem ao seu acervo tecnológico e informacional para otimizar seus processos e possuírem o domínio de dados estratégicos capazes de agregar valor e vantagem competitiva frente aos concorrentes, além de servir como instrumento básico para o aumento de sua produtividade (Rezende \& Abreu, 2008).

Não apenas a tecnologia evoluiu, mas também a profissão do Secretário Executivo, haja vista que esta categoria de profissionais passou por mudanças bastante significativas em seu perfil de atuação. Além da proficiência nas atividades técnicas executadas - tais como atendimento telefônico, controle e organização de agenda e reuniões, arquivamento de documentos etc. -, o profissional de Secretariado Executivo vem ganhando maior espaço nas organizações devido ao seu dinamismo (Mattos, 2000), sua iniciativa, polivalência, assessoria qualificada, participação como agente facilitador (Natalense, 1995), dentre outras competências. Para tanto, o profissional de Secretariado Executivo dispõe de instrumentos que contribuem para que seu trabalho seja realizado de modo mais otimizado, entre os quais os mais avançados recursos da tecnologia da informação (Azevedo \& Costa, 2000).

Este estudo tem como finalidade analisar a intensidade da utilização de diferentes categorias de aplicativos de TI pelos profissionais de Secretariado Executivo, verificando quais as ferramentas mais utilizadas, e quais aquelas que ainda demandam uma maior taxa de utilização; com o objetivo de viabilizar o aprimoramento operacional e maximização dos resultados organizacionais. O texto está dividido em cinco diferentes seções: esta introdução, apresentando a temática da pesquisa; a revisão de literatura, que realiza a apresentação dos conceitos teóricos que fundamentaram a classificação das diferentes categorias de sistemas de informação consideradas neste estudo; a metodologia, onde são demonstrados os procedimentos de análise quantitativa utilizados na investigação; a apresentação dos resultados, que aponta e efetua uma breve discussão dos principais resultados encontrados; e a conclusão, onde se apresentam os principais resultados obtidos e se tecem algumas considerações acerca desta pesquisa e das possibilidades de realização de novos estudos sobre o tema. 


\section{REVISÃO DE LITERATURA}

Os sistemas de informação constituem uma categoria específica entre os diferentes tipos de sistemas. Assim, podem ser definidos por um conjunto de determinadas caraterísticas, que os distinguem e os identificam como modelos especializados na coleta, processamento e fornecimento de informações. Além, disso estes sistemas podem ser classificados conforme diversas tipologias, sendo as principais: classificação por estrutura organizacional; por área funcional; por arquitetura do sistema de informação; e por tipo de suporte.

\subsection{CARACTERÍSTICAS DOS SISTEMAS DE INFORMAÇÃO}

Segundo Andrade, Audy e Cidral (2005), um sistema de informação deve produzir feedback (ou retroalimentação) a respeito das atividades operantes no sistema. O feedback promove um "mecanismo de controle de monitoria" a fim de que todos os processos do sistema sejam avaliados para que, então, se possa identificar se o sistema está cumprindo os objetivos para o qual foi designado. É competência do controle do sistema de informação determinar se as ações estão condizentes com o desempenho de padrão esperado; e é nesta etapa que os erros são identificados e corrigidos.

Contextualizando as atividades dos sistemas de informações, O’Brien (2003) ilustrou cada função sistêmica sob a análise a seguir: a entrada de dados pode ser feita, por exemplo, com cliques de acesso em websites e consultas em fóruns virtuais; o processamento pode ser identificado no momento em que algum colaborador executar em seu computador, programas que fazem parte dos recursos de software; a saída pode ser representada pela exibição em tela ou impressão de relatórios; o armazenamento está relacionado ao local onde os arquivos e informações são guardados (banco de dados, mídias externas - disco rígido, pen drive) e; como atividade de controle, pode-se citar o uso de senhas para acesso a programas e domínios.

Na Figura 1, Laudon e Laudon (2004) esquematizam o funcionamento das atividades de um sistema de informação. Nesta figura, é representado um sistema e o ambiente onde ele se encontra.

É observado que o feedback é direcionado a determinadas pessoas para que haja a apuração de entradas, obedecendo à lógica do controle. Já os fatores ambientais, como clientes e fornecedores, interagem com a organização e seu sistema.

Revista de Gestão e Secretariado - GeSec, São Paulo, v. 5, n. 2, p 138-157, mai./ago. 2014. 
Figura 1 - Funções de um Sistema de Informação.

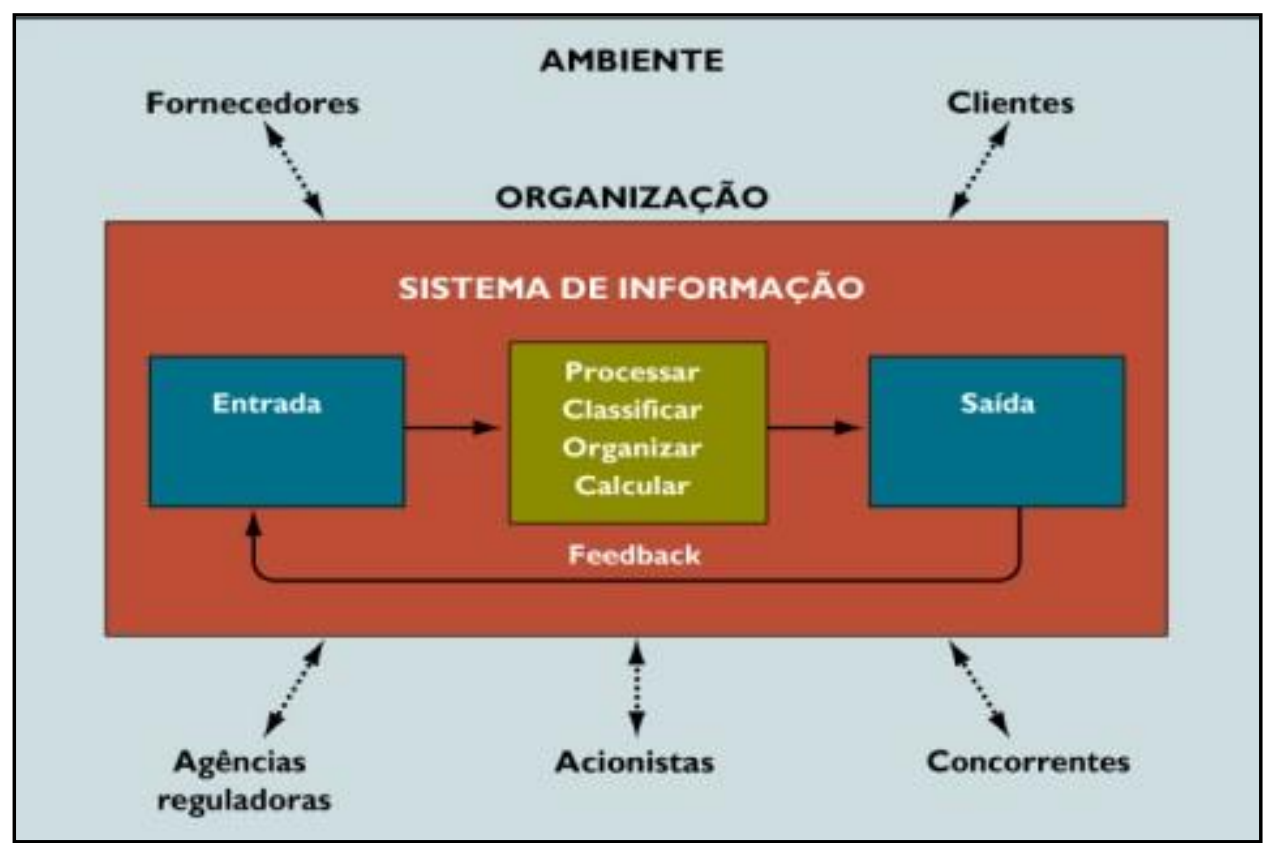

Fonte: Laudon \& Laudon, 2004, p. 8.

Para tal processamento e fornecimento de informações, os sistemas de informação são divididos em duas categorias: informais e formais. Os sistemas informais são alimentados por ações não declaradas, onde não há notificação do tipo e tampouco do modo que a informação será utilizada, como, por exemplo, as redes de fofocas. Os sistemas formais, ao contrário, são baseados em dados e processos aceitáveis, com regras definidas previamente e tendo por finalidade utilizar estes dados e informações gerados da melhor forma. A falta de flexibilidade é proposital justamente para que a estrutura não se perca e os dados sejam inseridos e armazenados corretamente. Estes sistemas de informação formais podem ser manuais ou informatizados. O modo manual utiliza a escrita convencional (lápis e caneta) e papel. Os sistemas informatizados, ao contrário, precisam de recursos tecnológicos como equipamentos e softwares para realizar o processamento e a difusão de informações (Laudon \& Laudon, 2004).

\subsection{CLASSIFICAÇÃO DOS SISTEMAS DE INFORMAÇÃO}

Os sistemas de informação, conforme Turban, McLean e Wetherbe (2004), são classificados em quatro maneiras: por estrutura organizacional, por área funcional, por arquitetura do sistema de informação e por tipo de suporte. Esses sistemas são baseados em computadores, portanto são compostos por hardware, software, dados, procedimentos e pessoas (usuários).

Revista de Gestão e Secretariado - GeSec, São Paulo, v. 5, n. 2, p 138-157, mai./ago. 2014. 
Os sistemas de informação classificados por estrutura organizacional são desenvolvidos para utilização conforme o esquema hierárquico da organização, distribuídos para departamentos, unidades operacionais e para colaboradores específicos, podendo ser independentes ou conectados entre si. Dentro desta classificação há os sistemas de informações por departamento, os quais realizam uma função específica para o usuário. Por exemplo, no Departamento de Gestão de Pessoas, pode-se utilizar este sistema para monitorar o ponto eletrônico dos colaboradores; os sistemas de informações empresariais formados por um conjunto de aplicativos departamentais e funcionais; e os sistemas interorganizacionais, responsáveis pela ligação entre diversas empresas, usados entre organizações parceiras em suas transações e no comércio eletrônico.

Sistemas de informação classificados por área funcional são compostos principalmente pelos sistemas nas áreas contábil, financeira, industrial, marketing e gestão de recursos humanos. Estes recebem o suporte dos sistemas de informação em nível departamental.

$\mathrm{Na}$ classificação de sistemas de informação por arquitetura, estes são responsáveis por "conceituar as necessidades de informação do negócio central da empresa, o que inclui definir de que forma tais necessidades serão supridas” (Turban; Mclean \& Wetherbe, 2004, p. 68).

Os sistemas de informação classificados por tipo de suporte são divididos nas seguintes categorias principais:

1) Sistema de processamento de transação (SIT), que tem por objetivo fornecer informações necessárias para manter de modo eficiente o negócio da empresa, sendo responsável por monitorar, coletar, armazenar, processar e disseminar a informação para as transações repetitivas e rotineiras, como registrar todas as contas a pagar e a receber, os níveis de estoque, dentre outras atividades (Turban; Mclean \& Wetherbe, 2004).

2) Sistema de informação gerencial (SIG) ou sistema tático, que fornece suporte às atividades de planejamento, organização e controle empresarial dentro de um curto prazo de tempo, oferecendo informações necessárias para o processo de decisão. Assim como os sistemas de processamento de transações, os SIGs computadorizados são programados para compilar os dados e preparar relatórios, isto somado à tarefa de conceder respostas rápidas aos seus usuários. Além de relatórios estatísticos, de exceções, periódicos e especiais, os sistemas de informação gerenciais fornecem análises comparativas em relação tanto ao desempenho anterior quanto aos concorrentes, projeções negociais, detecção precoce de problemas, 
suporte às decisões rotineiras e opções de conexão estendidos a outras empresas, como correio eletrônico e sistemas de mensagens (Turban; Mclean \& Wetherbe, 2004).

3) Sistema de administração do conhecimento (KMS), que disponibiliza informações especializadas "que fazem parte da memória da empresa e que normalmente existem dentro de uma forma não estruturada" para toda a organização, "permitindo a resolução eficaz e eficiente de problemas, aprendizado dinâmico, planejamento estratégico e tomada de decisão" (Turban; Mclean \& Wetherbe, 2004, p. 326).

4) Sistema de automação de escritório (SAE), que tem por finalidade aumentar a produtividade dos trabalhadores de informação dentro da organização por meio de qualquer aplicação de Tecnologia da Informação. Estes colaboradores dividem-se em trabalhadores de dados, processando e disseminando informações, como secretários e contadores; e trabalhadores de conhecimento, "que criam conhecimento e informação" (Laudon \& Laudon, 2004, p. 326) como projetistas e arquitetos. As atividades que podem ser apoiadas pelos sistemas de automação de escritório são, por exemplo, edição de texto, editoração eletrônica, digitalização de documentos, utilização de banco de dados em computadores de mesa e planilhas eletrônicas (Turban; Mclean \& Wetherbe, 2004).

5) Sistema de apoio a decisões (SAD), que é baseado em computador, combinando modelos e dados empresariais a fim de solucionar problemas semiestruturados juntamente com o usuário. As maiores razões para que os gerentes concordem em possuir SADs nas organizações remetem à necessidade de obter informações rapidamente, atualizadas e precisas; nas dificuldades de ordem financeira e pela concorrência; e para atender as mais diversas questões imediatas da empresa. Não há um consenso sobre o que constitua um sistema de apoio à decisão, entretanto a maioria destes possui como atributos o suporte à tomada de decisão em todos os níveis gerenciais, combinando a capacidade de julgamento com informações objetivas; apoio a decisões independentes entre si ou sequenciais; utiliza métodos quantitativos; e com o decorrer do tempo, pode ser adaptado para lidar com modificações de condições (Turban; Mclean \& Wetherbe, 2004).

6) Sistema de informação empresarial (EIS), que atende as necessidades dos altos executivos da empresa, proporcionando rápido acesso à informação desejada e aos relatórios gerenciais. O EIS utiliza recursos como o drill down, que oferece aos usuários, ricos detalhes sobre qualquer informação requerida; os fatores críticos de sucesso que podem ser estratégicos, gerenciais ou operacionais e que podem estar presentes em toda a empresa, em um setor específico ou mesmo ter que ser considerado em cada colaborador para, então, serem

Revista de Gestão e Secretariado - GeSec, São Paulo, v. 5, n. 2, p 138-157, mai./ago. 2014. 
monitorados conforme indicadores de desempenho; e análises de tendências internas (dentro da empresa) e externas (mercado) utilizando modelos de previsão (Turban; Mclean \& Wetherbe, 2004).

7) Sistema de apoio a grupos (GSS) por meio do sistema de apoio à decisão em grupos (SADG), que oferece suporte aos colaboradores que trabalham coletivamente dentro da organização, uma vez que as decisões são tomadas, muitas vezes, em um processo horizontal, apesar da hierarquia empresarial. A exemplo dessa situação, pode-se citar uma decisão que deva ser tomada pelos gerentes de todas as áreas da organização. O SAD-G é um sistema que visa facilitar a resolução de problemas semi ou não estruturados que dependam de um grupo de pessoas, agilizando o processo decisório e melhorando a qualidade deste (Turban; Mclean \& Wetherbe, 2004). DeSanctis e Gallupe (1987) apontam como características principais desde sistema a facilidade de aprendizado e utilização que os usuários podem ter; é criado com um objetivo específico e programado tanto para um tipo de problema quanto para uma grande diversidade de decisões a serem tomadas pelo grupo; estimula ideias, liberdade de expressão e capacidade para resolução de conflitos; e é dotado de mecanismos que desestimulam reações negativas por parte do grupo. No que diz respeito à utilização de SAD-G em reuniões, a maioria das aplicações apoiam reuniões virtuais, devido à praticidade.

8) sistema de suporte inteligente interage consideravelmente mais com os profissionais do conhecimento em relação aos demais grupos de colaboradores e possui como tecnologia principal o Sistema Especialista (SE). O software de SE proporciona resoluções para os problemas empresariais a partir de um banco de dados com informações específicas sobre determinada área. Essas informações são previamente coletadas por profissionais especialistas ou de fontes documentais e transferidas para o computador. Ao acessar o sistema para consultar questões específicas, o SE faz deduções e propõe uma solução para o usuário, apresentando, se necessário, a lógica utilizada para a resolução tomada. Os maiores benefícios que os sistemas especialistas oferecem são: o aumento de qualidade das decisões, reduzindo o índice de erros; a confiabilidade gerada é grande, pois cada detalhe da circunstância levantada é levado em consideração; a redução do tempo na tomada de decisão; e até mesmo o treinamento que os colaboradores recebem deste sistema, por sua capacidade de explicação, gerando conhecimento organizacional (Turban; Mclean \& 
Wetherbe, 2004).

A essência de todos esses sistemas, direta ou indiretamente, é a comunicação por proporcionar intercâmbio e compartilhamento de informações. O Quadro 1 apresenta um resumo sintético dos sistemas de informação classificados por tipo de suporte. Os principais fatores utilizáveis das tecnologias para dar suporte à comunicação são as pessoas; a natureza das fontes e destinos, que incluem pessoas, sensores, e bancos de dados; a localização do destinatário da mensagem; o tempo, caso a mensagem deva ser programada para um horário e recebida logo em seguida ou após algum tempo; e os meios de comunicação, envolvendo mídias como texto e áudio (Turban; Mclean \& Wetherbe, 2004).

\begin{tabular}{|c|c|}
\hline Sistema de processamento de transação (SIT) & $\begin{array}{l}\text { Realização de transações repetitivas e rotineiras (contas a } \\
\text { pagar, controle de estoque etc.) }\end{array}$ \\
\hline Sistema de informação gerencial (SIG) & $\begin{array}{l}\text { Suporte às atividades de gestão empresarial (planejamento, } \\
\text { organização e controle) }\end{array}$ \\
\hline $\begin{array}{l}\text { Sistema de administração do conhecimento } \\
\text { (KMS) }\end{array}$ & Disponibilização de informações especializadas \\
\hline Sistema de automação de escritório (SAE) & $\begin{array}{l}\text { Aumento da produtividade dos usuários nas rotinas } \\
\text { administrativas (edição de textos, planilhas eletrônicas etc.) }\end{array}$ \\
\hline Sistema de apoio a decisões (SAD) & Apoio à solução de problemas semiestruturados \\
\hline Sistema de informação empresarial (EIS) & $\begin{array}{l}\text { Acesso a relatórios com indicadores de desempenho e análises } \\
\text { de tendências }\end{array}$ \\
\hline Sistema de apoio a grupos (GSS) & Suporte a realização de atividades e decisões coletivas \\
\hline Sistema especialista (SE) & $\begin{array}{l}\text { Soluções para problemas empresariais em áreas } \\
\text { específicas }\end{array}$ \\
\hline
\end{tabular}

Quadro 1 - Funções básicas dos sistemas de informação (classificados por tipo de suporte).

Fonte: Elaboração dos autores (2012). Adaptado de Turban; McLean \& Wetherbe (2004).

DeSanctis e Gallupe (1987) defendem a ideia de que para que haja uma estrutura de um sistema de comunicação, esta deva ser dividida em quatro partes, relacionando-se às pessoas: mesmo tempo/mesmo lugar, mesmo tempo/lugar diferente, tempo diferente/mesmo lugar e tempo diferente/lugar diferente. As empresas têm como necessidade comunicar-se eficazmente em todos os níveis hierárquicos a fim de que as informações sejam transmitidas de modo claro e objetivo, agilizando a tomada de decisões e os processos organizacionais. Sendo assim, as empresas utilizam meios de comunicação que facilitam a interação entre os colaboradores. Dentre esses meios estão o correio eletrônico, as mensagens instantâneas e a comunicação eletrônica de voz. 


\section{METODOLOGIA}

Trata-se de um estudo de natureza aplicada, uma vez que é realizada uma pesquisa com a finalidade de produzir um conhecimento que leve a fins práticos (Barros \& Lehfeld, 2000). A abordagem é de caráter quantitativo, ou seja, a análise dos dados resulta em informações estatisticamente comprovadas que representam um resultado generalizado da amostragem (Neves, 1996). Os procedimentos técnicos desta pesquisa envolvem os tipos documental e bibliográfico, fazendo uso de materiais já publicados, principalmente de livros e artigos; e pesquisa de campo, onde ocorre a observação dos fatos, sem intervenções. Não há como manipular as variáveis, somente percebê-las e analisá-las (Gil, 1991; Rodrigues, 2007).

O universo da pesquisa consiste nos profissionais graduados no curso de Bacharelado em Secretariado Executivo Bilíngue - egressos da Universidade da Amazônia (Unama) e Secretariado Executivo Trilíngue, egressos da Universidade do Estado do Pará (Uepa). As exigências para compor o perfil dos investigados foram: estar exercendo atividades como Secretário Executivo e residir na cidade de Belém, no estado do Pará. Por não ser possível ter acesso ao número exato de profissionais graduados no curso de Secretariado Executivo que no momento atuam na área, em função da falta de dados estatísticos de origem confiável, optou-se por utilizar uma amostra do tipo não probabilística (Cooper \& Schindler, 2003) e acidental (Silva \& Menezes, 2001), uma vez que os integrantes foram indicados de modo aleatório por colaboradores da pesquisa, totalizando o tamanho da amostra em 35 profissionais.

Os dados para este estudo foram coletados por meio de questionários aplicados no período de julho a novembro de 2011, compostos por duas séries de questões. A primeira série objetiva investigar o caráter sociodemográfico e profissional da amostra, e a segunda série, de caráter comportamental, é composta por uma tabela e utiliza como critério para resposta a escala Likert (Johnson \& Wichern, 2002). As perguntas possuem respostas de múltipla escolha referenciando-se ao grau de utilização dos recursos de Tecnologia da Informação.

As perguntas comportamentais foram divididas em quatro grupos de recursos tecnológicos com base na classificação de Sistemas de Informação dada por Turban, McLean e Wetherbe (2004), sendo estes: Automação de Escritório (sistemas de automação de escritório); Comunicação (sistemas de comunicação); Gestão de Processos que, nesta pesquisa, refere-se ao fluxo de informações e documentos (sistemas de apoio a grupos); e Softwares Empresariais (sistemas de

Revista de Gestão e Secretariado - GeSec, São Paulo, v. 5, n. 2, p 138-157, mai./ago. 2014. 
processamento de transação). Foram adotadas cinco categorias para a mensuração dos dados: (0) não sabe utilizar; (1) não sabe utilizar, mas está em treinamento; (2) utiliza apenas parcialmente a ferramenta; (3) utiliza a maior parte dos recursos da ferramenta; (4) utiliza todos os recursos da ferramenta.

Para a análise estatística descritiva dos dados coletados foram utilizados os seguintes métodos: Distribuição de Frequências, Análise de Desvio Padrão e Cálculo de Índices. A análise da distribuição de frequências permite a construção de gráficos unidimensionais utilizados para a caracterização da amostra, que permitiu a caracterização do perfil sociodemográfico e profissional dos respondentes. O cálculo dos desvios-padrões foi realizado com a finalidade de permitir a análise do índice de dispersão das respostas, e assim verificar a possibilidade de agrupamento dos indivíduos em diferentes grupos homogêneos, por meio da Análise de Cluster (Hair; Anderson; Tathan, \& Black, 2005). O cálculo dos índices teve como objetivo determinar o grau de conhecimento das ferramentas de TI por parte de cada integrante da amostra, assim como determinar o grau de conhecimento conjunto da amostra em relação a cada variável. Para isto, foi utilizada a seguinte fórmula matemática:

$$
(\mathrm{n} 4 \times 1+\mathrm{n} 3 \times 0,75+\mathrm{n} 2 \times 0,50+\mathrm{n} 1 \times 0,25) / \mathrm{N}
$$

Onde $\mathbf{n}=$ número de variáveis observadas, para o cálculo dos índices individuais; e $\mathbf{N}=$ número de observações (indivíduos integrantes da amostra), para o cálculo dos índices coletivos. Para análise dos resultados foram considerados os seguintes valores: (a) 0,000 a 0,399: ruim; (b) 0,400 a 0,699: regular; e (c) 0,700 a 1,000: bom.

A Análise de Cluster tem por finalidade classificar e agrupar indivíduos em função de sua similaridade diante de uma determinada característica ou de um conjunto destas. Esses grupos objetivam reunir os elementos em conjuntos definidos por características específicas de seus integrantes, de modo que seja possível observar a variação mínima intragrupo e a variação máxima intergrupos. Nesta pesquisa, foi utilizado o procedimento hierárquico aglomerativo, em que os elementos, tomados separadamente, são progressivamente combinados, em função de sua similaridade, de forma a construir novos agrupamentos até que se obtenha um único agrupamento (Hair et al., 2005). Foi empregado o método de Ward, calculado a partir da medida de dissimilaridade da distância euclidiana até o quadrado entre dois elementos (i e k), representado pela expressão (Mingoti, 2005):

Revista de Gestão e Secretariado - GeSec, São Paulo, v. 5, n. 2, p 138-157, mai./ago. 2014. 


$$
I D C_{i}=\sum_{j=1}^{q}\left[\frac{\lambda_{\mathrm{j}}}{\sum_{j} \lambda_{\mathrm{j}}} F P_{i j}\right](i=1,2, \ldots, n)
$$

Onde dij2 representa a j-ésima característica do i-ésimo indivíduo; xik representa a j- ésima característica do i-ésimo indivíduo; e, xik' representa a j-ésima característica do i'-esimo indivíduo. Para as análises estatísticas, foram utilizados os seguintes aplicativos: a planilha eletrônica MS Excel 2010 e o pacote computacional SPSS Statistics 17.0.

\section{APRESENTAÇÃO E DISCUSSÃO DOS RESULTADOS}

A apresentação dos resultados do estudo está dividida em três etapas. A caracterização da amostra define o perfil dos indivíduos que fizeram parte da pesquisa, assim como as características das organizações em que estes profissionais atuavam na época do estudo. A análise descritiva apresenta os índices estatísticos que apontam o grau de utilização das diferentes ferramentas de TI pelos profissionais de Secretariado Executivo, assim como os índices médios de cada um dos grupos ou categorias de softwares, definidos conforme os critérios da pesquisa. Em seguida, são apresentados os resultados da análise de cluster, por meio da qual se pode classificar em integrantes da amostra em grupos, conforme as médias dos índices de utilização das categorias de softwares.

\subsection{CARACTERIZAÇÃO DA AMOSTRA}

A amostra utilizada neste estudo é composta por 35 profissionais graduados em Secretariado Executivo, em cursos superiores da Universidade do Estado do Pará (Uepa) e da Universidade da Amazônia (Unama). O grupo é integrado majoritariamente por indivíduos do sexo feminino (83\%), com idade entre 19 e 30 anos (77\%), solteiros (71\%), e com renda familiar entre R\$1.610,00 e R\$ 6.940,00 (77\%), equivalente à classe C, segundo os critérios de classificação socioeconômica do Instituto Brasileiro de Geografia e Estatística (IBGE) à época da realização da pesquisa.

Quanto às organizações em que atuavam estes profissionais, destacam-se o setor público $(57 \%)$ e o setor de serviços $(26 \%)$. Em relação à forma de constituição, a maioria caracteriza-se

Revista de Gestão e Secretariado - GeSec, São Paulo, v. 5, n. 2, p 138-157, mai./ago. 2014. 
como empresa pública (34\%) ou órgão público (34\%). A grande maioria dos indivíduos declarou trabalhar em organizações de grande porte (74\%), com mais de 200 funcionários, conforme os critérios do Serviço de Apoio às Micro e Pequenas Empresas (Sebrae).

\subsection{ANÁLISE DESCRITIVA}

$\mathrm{Na}$ análise descritiva, os softwares foram agrupados em quatro conjuntos de ferramentas, a partir da classificação de Sistemas de Informação elaborada por Turban, McLean e Wetherbe (2004). São apresentados os índices de utilização de cada software e o índice de cada grupo, representado pela média aritmética dos índices dos softwares integrantes de cada grupo (Tabela 1). Observa-se que o grupo de softwares de Comunicação obteve o maior índice de utilização pelos indivíduos da pesquisa $(0,704)$, bastante próximo ao índice obtido pelos softwares de Automação de Escritório (0,682). Estes resultados corroboram as informações dos autores supracitados, no sentido de que a sua aplicação contribui para um significativo aumento da produtividade dos trabalhadores. Por outro lado, os grupos de Gestão de Processos (0,566) e Softwares Empresariais $(0,550)$, obtiveram índices bastante inferiores, o que denota um grau significativamente mais baixo de utilização pelos Secretários Executivos em suas atividades profissionais. Neste caso, os resultados do estudo apontam para uma possível perda de eficiência no trabalho dos usuários nas organizações, pois estas categorias de sistemas são responsáveis pelo fornecimento de informações indispensáveis para a maximização da produtividade da empresa, assim como são importantes ferramentas de suporte ao trabalho coletivo (Turban; McLean \& Wetherbe, 2004).

Tabela 1- Índices de Utilização das Ferramentas de TI

\begin{tabular}{|l|l|l|l|l|l|c|c|}
\hline \multicolumn{1}{|c|}{ FERRAMENTAS DE TI } & & & & & Índice & DP* \\
\hline \multicolumn{1}{|c|}{ AUTOMAÇÃO DE ESCRITÓRIO } & & & & & & $\mathbf{0 , 6 8 2}$ & $\mathbf{0 , 7 5 2}$ \\
\hline Processador de Texto (Word, Writer etc.) & & & & & & 0,921 & 1,063 \\
\hline Planilha Eletrônica (Excel, Calc etc.) & & & & & & 0,700 & 0,700 \\
\hline Apresentação Gráfica (Power Point, Impress etc.) & & & & & & 0,729 & 0,755 \\
\hline Banco de Dados (Access etc.) & & & & & & 0,379 & 0,490 \\
\hline \multicolumn{1}{|c|}{ COMUNICAÇÃo } & & & & & & $\mathbf{0 , 7 0 4}$ & $\mathbf{0 , 7 3 3}$ \\
\hline Correio Eletrônico (Outlook etc.) & & & & & & 0,907 & 1,046 \\
\hline Mensagens Instantâneas (MSN, Messenger etc.) & & & & & & 0,836 & 0,869 \\
\hline Chamadas Telefônicas (Skype etc.) & & & & & & 0,657 & 0,579 \\
\hline Videoconferência & & & & & & 0,414 & 0,436 \\
\hline \multicolumn{1}{|c|}{ GESTÃO DE PROCESSOS } & & & & & & $\mathbf{0 , 5 6 6}$ & $\mathbf{0 , 6 2 1}$ \\
\hline Gerenciamento de Documentos (Notes etc.) & & & & & & 0,557 & 0,464 \\
\hline Gerenciamento de Projetos (Project etc.) & & & & & & 0,236 & 0,768 \\
\hline
\end{tabular}

Revista de Gestão e Secretariado - GeSec, São Paulo, v. 5, n. 2, p 138-157, mai./ago. 2014. 


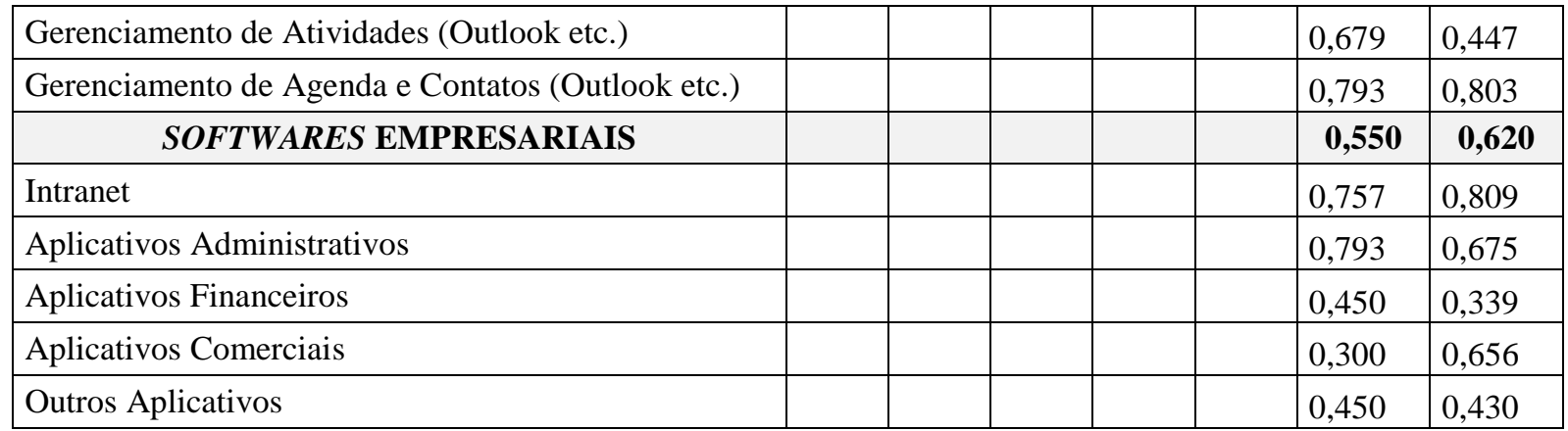

*DP: Desvio padrão

Fonte: Resultados da pesquisa (2012).

Entre os softwares de Automação de Escritório, nota-se um elevado índice de utilização de processadores de texto $(0,921)$, enquanto as ferramentas eletrônicas para gerenciamento de Bancos de Dados são muito pouco utilizadas $(0,379)$. Entre os softwares de Comunicação, o destaque positivo é para ferramentas de Correio Eletrônico $(0,907)$, ao passo que as ferramentas para Videoconferência registram um índice bem menor $(0,414)$. No grupo dos softwares de Gestão de Processos, observa-se um elevado índice de utilização de ferramentas de Gerenciamento de Agenda e Contatos $(0,793)$, enquanto as ferramentas eletrônicas para Gerenciamento de Projetos registraram o pior resultado em todo o estudo $(0,236)$. Por fim, entre os chamados Softwares Empresariais, o melhor resultado foi para os Aplicativos Administrativos (0,793), enquanto os Aplicativos Comerciais obtiveram o resultado mais baixo $(0,300)$.

Os resultados da análise descritiva indicam claramente duas grandes aplicações dos recursos de TI pelos profissionais de Secretariado Executivo que fizeram parte do estudo: por um lado, a edição de textos, para a impressão e guarda eletrônica de documentos escritos; e por outro lado, das ferramentas de comunicação eletrônica, com maior destaque para o correio eletrônico. Estas informações demonstram que a grande maioria dos recursos das ferramentas disponíveis para as empresas ainda é bastante subutilizado por estes profissionais, o que implica que grande parte dos consideráveis ganhos de produtividade proporcionados por estes recursos ainda não está sendo plenamente aproveitada pelas organizações e pelos profissionais que deveriam utilizá-los. Segundo as referências utilizadas na pesquisa, a utilização intensificada dos aplicativos de automação de escritórios e de comunicação favorece a elevação da produtividade dos usuários (Turban; McLean \& Wetherbe, 2004), porém, a subutilização das demais categorias de sistemas implica no desperdício de boa parte dos ganhos de competitividade que seriam possibilitados por estes softwares.

Revista de Gestão e Secretariado - GeSec, São Paulo, v. 5, n. 2, p 138-157, mai./ago. 2014. 


\subsection{ANÁLISE DE CLUSTER}

Johnson e Wichern (2002) afirmam que não há um procedimento padrão para selecionar a quantidade de clusters. Assim, optou-se pela definição do número de clusters por meio da "regra de parada". De acordo com este procedimento, devem-se utilizar as medidas de similaridade. No momento em que se verificar uma variação desproporcional nos coeficientes de proximidade, a solução imediatamente anterior pode ser utilizada para que se defina o número de agrupamentos (Tabela 2). Assim, será selecionada a opção com a maior similaridade interna e a maior diferença externa entre os grupos (Hair et al., 2005). Neste caso, tal procedimento teve como resultado a indicação de três agrupamentos.

Tabela 2 - "Regra de Parada"

\begin{tabular}{|l|l|l|l|}
\hline \multirow{2}{*}{\begin{tabular}{c}
\multirow{2}{*}{$\begin{array}{c}\mathbf{N}^{\mathbf{0}} \text { de } \\
\text { clusters }\end{array}$} \\
Cling
\end{tabular}} & \multirow{2}{*}{ Coeficiente } & \multicolumn{2}{c|}{ Variaço } \\
\cline { 3 - 4 } & & Absoluta & \% \\
\hline 6 & 2,673 & 0,325 & $32,47 \%$ \\
\hline 5 & 3,277 & 0,604 & $60,41 \%$ \\
\hline 4 & 3,967 & 0,690 & $69,04 \%$ \\
\hline 3 & 4,660 & 0,693 & $69,30 \%$ \\
\hline 2 & 5,958 & 1,298 & $129,78 \%$ \\
\hline 1 & 8,958 & 3,000 & $299,98 \%$ \\
\hline
\end{tabular}

Fonte: Resultados da Pesquisa (2012).

A aplicação da regra de parada indicou que a amostra pode ser classificada em três agrupamentos, indicando uma variação absoluta desproporcional na segunda etapa do procedimento. No primeiro grupo, foram classificados 13 profissionais, representando 37,14\% da amostra pesquisada. O segundo grupo reúne 12 profissionais, equivalentes a 34,29\% da amostra. Já o terceiro agrupamento é integrado por 10 profissionais e representa 28,57\% da amostra. A análise dos índices médios das variáveis analisadas permite a observação do padrão de comportamento predominante em cada agrupamento (Tabela 3).

Observa-se que o primeiro grupo obteve uma média geral de utilização dos aplicativos de índice 0,484, considerada regular (entre 0,400 e 0,699). Seu melhor desempenho foi nos aplicativos de escritório, com índice 0,591, e o pior foi nos aplicativos de processos, com índice 0,356. Desta maneira, o agrupamento passou a ser denominado "usuários regulares de aplicativos de escritório". O segundo grupo obteve o melhor índice médio geral, com 0,714. O melhor resultado deste agrupamento também foi nos aplicativos de escritório, com 0,833, e o seu pior resultado foi nos

Revista de Gestão e Secretariado - GeSec, São Paulo, v. 5, n. 2, p 138-157, mai./ago. 2014. 
aplicativos de processos, com índice 0,589. Assim, este agrupamento recebeu a denominação de "usuários intensivos de aplicativos de escritório". Segundo Turban, McLean e Wetherbe (2004), estes sistemas (aplicativos de escritório) contribuem significativamente para a elevação da produtividade de trabalhadores da informação dentro das organizações. Conforme Laudon e Laudon (2004) são sistemas tipicamente utilizados por funcionários que realizam o processamento e disseminação de informações, como os secretários executivos.

Em relação ao terceiro grupo, observa-se que o seu melhor resultado foi nos aplicativos de processos, com índice 0,813, e o pior nos softwares empresariais, com índice 0,600. Este agrupamento passou então a ser denominado "usuários intensivos de aplicativos de processos". Para Turban, McLean e Wetherbe (2004) esta categoria de aplicativos favorece a atuação das equipes nas organizações, enquanto DeSanctis e Gallupe (1987) destacam a sua facilidade de utilização e o estímulo à criatividade dos usuários.

Tabela 3 - Índices médios dos clusters

\begin{tabular}{|l|c|c|c|c|c|}
\hline \multirow{2}{*}{ Categorias de usuários } & \multicolumn{5}{c|}{ Categorias de aplicativos } \\
\cline { 2 - 6 } & Escritório & Comunicação & Processos & Empresarial & Média \\
\hline $\begin{array}{l}\text { Regulares de aplicativos de } \\
\text { escritório }\end{array}$ & 0,591 & 0,582 & 0,356 & 0,408 & 0,484 \\
\hline $\begin{array}{l}\text { Intensivos de aplicativos de } \\
\text { escritório }\end{array}$ & 0,833 & 0,771 & 0,589 & 0,663 & 0,714 \\
\hline $\begin{array}{l}\text { Intensivos de aplicativos de } \\
\text { processos }\end{array}$ & 0,619 & 0,781 & 0,813 & 0,600 & 0,703 \\
\hline Média & 0,681 & 0,711 & 0,586 & 0,557 & 0,634 \\
\hline
\end{tabular}

Fonte: Resultados da pesquisa (2012).

Analisando os resultados obtidos pelas diferentes categorias de aplicativos, pode-se verificar que os aplicativos de comunicação obtiveram o melhor índice médio geral, com 0,711. O índice mais baixo ficou com os aplicativos empresariais, com resultado de 0,557. O melhor índice médio em um agrupamento foi dos aplicativos de escritório no grupo de usuários intensivos de aplicativos de escritório, com resultado de 0,833; enquanto o pior resultado médio em um agrupamento foi dos aplicativos de processos no grupo dos usuários regulares de aplicativos de escritório, com índice de 0,356.

Observando-se os resultados médios dos agrupamentos, verifica-se que o grupo dos usuários regulares de aplicativos de escritório obteve um índice médio regular $(0,484)$, enquanto os grupos de usuários intensivos de aplicativos de escritório e os usuários intensivos de aplicativos

Revista de Gestão e Secretariado - GeSec, São Paulo, v. 5, n. 2, p 138-157, mai./ago. 2014. 
de processos obtiveram resultados considerados bons (0,714 e 0,703). Porém, quando se analisam os resultados das categorias de aplicativos, observa-se que somente os aplicativos de comunicação obtiveram um índice médio bom $(0,711)$, enquanto os aplicativos de escritório, de processos e empresariais obtiveram índices médios somente regulares (0,681 para o primeiro, 0,586 para o segundo e 0,557 para o terceiro). Desta maneira, o índice médio geral de todos os resultados, considerando-se tanto os agrupamentos quanto as categorias de aplicativos também pode ser considerado como regular $(0,634)$. Este resultado revela que, embora alguns resultados específicos de agrupamentos e aplicativos tenham sido classificados como bons (acima de 0,700), o resultado geral obtido pela amostra revela-se apenas mediano (entre 0,400 e 0,699).

\section{CONSIDERAÇÕES FINAIS}

Os resultados da análise descritiva demonstram que a categoria dos aplicativos de comunicação é a mais utilizada pelos profissionais integrantes da amostra pesquisada, com um índice médio de 0,704, considerado bom. Porém, o melhor resultado de um aplicativo específico foi o dos Processadores de Texto, com índice 0,921. Por outro lado, a categoria de aplicativos empresariais obteve um índice médio de apenas 0,550, enquanto os aplicativos de gerenciamento de projetos foram os softwares específicos com o pior resultado, com um índice de apenas 0,236.

Ao se realizar o agrupamento dos indivíduos por meio da análise de cluster, observa-se que a amostra pode ser classificada em três grupos relativamente homogêneos. O grupo classificado como usuários regulares de aplicativos de escritório, com 37,14\% dos indivíduos, apresenta um índice médio geral de 0,484 (regular). O grupo definido como usuários intensivos de aplicativos de escritório, que reúne 34,29\% dos indivíduos, apresenta um índice médio geral de 0,714, considerado bom e o melhor resultado entre os agrupamentos. Já o grupo denominado usuários intensivos de aplicativos de processos, com 28,57\% dos integrantes da amostra, registra um índice médio geral de 0,703, também considerado bom. Entre as categorias de aplicativos, somente os softwares de comunicação obtiveram um bom resultado, com um índice médio de 0,711 . Os demais obtiveram resultados considerados regulares. $\mathrm{O}$ índice médio geral da pesquisa, considerando-se tanto os agrupamentos quanto as categorias de aplicativos, foi considerado como regular, resultado de 0,634.

Uma análise desses resultados estatísticos demonstra que há uma utilização desproporcional dos recursos da tecnologia da informação por parte dos profissionais de

Secretariado Executivo integrantes da amostra pesquisada. Enquanto aplicativos específicos,

Revista de Gestão e Secretariado - GeSec, São Paulo, v. 5, n. 2, p 138-157, mai./ago. 2014. 
como os processadores de texto, são de larga utilização, ferramentas extremamente úteis para esta classe profissional, como os softwares de gerenciamento de projetos, são quase ignorados. Verificase então que a grande maioria dos profissionais pesquisados concentra seu uso desses recursos em um pequeno número de aplicativos, desperdiçando-se o potencial de aplicativos com grande poder de operação em atividades como o processamento e o gerenciamento de bancos de dados e a gestão de projetos, atividades bastante presentes na rotina desta classe profissional.

A se considerar os resultados deste estudo, torna-se mister a criação de programas de qualificação acadêmica e profissional destinados a promover uma otimização do uso das ferramentas de tecnologia da informação, particularmente no caso de recursos tecnológicos destinados ao gerenciamento de projetos e à gestão de bancos de dados. Também preocupa a pequena taxa de utilização dos chamados aplicativos empresariais, que são softwares específicos relacionados a atividades operacionais próprias de cada organização. Tal fato pode indicar que os secretários executivos possam estar sendo subutilizados e desperdiçando parte de sua capacidade de contribuição para os objetivos organizacionais.

Ressalte-se que este estudo teve caráter meramente exploratório, tornando-se necessários estudos de natureza descritiva e explicativa para que se possa compor um painel mais completo dos resultados inicialmente detectados. Recomenda-se então o aprofundamento deste tipo de pesquisa, com a finalidade de obter um maior detalhamento das informações obtidas, determinar que fatores são responsáveis pelos resultados observados, e eventualmente, propor estratégias para a superação dos problemas porventura confirmados por meio deste aprofundamento investigativo.

Revista de Gestão e Secretariado - GeSec, São Paulo, v. 5, n. 2, p 138-157, mai./ago. 2014. 


\section{REFERÊNCIAS}

Andrade, Gilberto; Audy, Jorge \& Cidral, Alexandre (2005). Fundamentos de sistema de informação. Porto Alegre: Bookman.

Azevedo, Ivanize \& Costa, Sylvia Ignácio (2000). Secretária: um guia prático. São Paulo: Senac.

Barros, Aidil \& Lehfeld (2000). Fundamentos de metodologia (2a ed.). São Paulo: Makron Books.

Cooper, Donald R. \& Schindler, Pamela S. (2003). Métodos de pesquisa em administração (7a ed.). Porto Alegre: Bookman.

Desanctis, G. \& Gallupe, B. (1987). A Foundation for the study of group decision support systems. Management Science, vol. 33, nº 5, 589-609.

Gil, Antônio Carlos (1991). Como elaborar projetos de pesquisa. São Paulo: Atlas.

Hair, J.; Anderson, R. E.; Tathan, R. L.\& Black, W. C. (2005). Análise multivariada de dados (5a ed.) Porto Alegre: Bookman.

Johnson, R. A. \& Wichern, D. W. (2002). Applied multivariate statistical analysis (5a ed.). New Jersey: Prentice Hall.

Laudon, Jane P \& Laudon, Kenneth (2004). Sistemas de informação gerenciais (5a ed.). São Paulo: Pearson Prentice Hall.

Mattos, Vera (2000). Super secretária (2a ed.) São Paulo: Nobel.

Mingoti, S. A. (2005). Análise de dados através de métodos de estatística multivariada: uma abordagem aplicada. Belo Horizonte: Editora UFMG.

Natalense, M. L. C. (1995). Secretária executiva: manual prático. São Paulo: IOB.

Neves, J. L. (1996). Pesquisa qualitativa: características, usos e possibilidades. Cadernos de Pesquisas em Administração (vol. 1, n. 3, $2^{\circ}$ sem.).

O’Brien, James (2003). Sistemas de Informação e as decisões gerenciais na era da Internet (2a ed.) São Paulo: Saraiva.

Rezende, D. A. \& Abreu, A. F. (2008). Tecnologia da informação aplicada a sistema de informações empresariais (5a ed.). São Paulo: Atlas.

Revista de Gestão e Secretariado - GeSec, São Paulo, v. 5, n. 2, p 138-157, mai./ago. 2014. 
Rodrigues, William Costa (2007). Metodologia científica. Rio de Janeiro: Instituto Superior de Tecnologia de Paracambi. Recuperado em 17 abril (2011), de <http://professor.ucg.br/siteDocente/admin/arquivosUpload/3922/material/Willian\%20Costa\%20 Rodrigues_metodologia_cientifica.pdf $>$.

Silva, Edna Lúcia da \& Menezes, Estera Muszkat (2001). Metodologia da pesquisa e elaboração da dissertação (3a ed. rev. atual.). Florianópolis: Laboratório de Ensino a Distância da UFSC.

Turban, Efraim; Mclean, Ephraim \& Wetherbe, James (2004). Tecnologia da informação para gestão: transformando os negócios na economia digital (3a ed.). Porto Alegre: Bookman. 